\title{
Molecular profiling to identify molecular mechanism in esophageal cancer with familial clustering
}

\author{
INDRANIL CHATTOPADHYAY ${ }^{1}$, RUPKUMAR PHUKAN ${ }^{3}$, AVNINDER SINGH $^{1}$, \\ MADAVAN VASUDEVAN ${ }^{4}$, JOYDEEP PURKAYASTHA ${ }^{2}$, STEPHEN HEWITT $^{5}$, \\ AMAL KATAKI $^{2}$, JAGADISH MAHANTA ${ }^{3}$, SUJALA KAPUR ${ }^{1}$ and SUNITA SAXENA ${ }^{1}$ \\ ${ }^{1}$ Institute of Pathology, New Delhi-110029; ${ }^{2}$ Dr B. Borooah Cancer Institute, Guwahati-781016, Assam; \\ ${ }^{3}$ Regional Medical Research Center, Dibrugarh-786001, Assam; ${ }^{4}$ Genotypic Technology [P] Ltd, 112 , \\ Bangalore-560001, India; ${ }^{5}$ Laboratory of Pathology, National Cancer Institute, Bethesda, MD 20892, USA
}

Received November 5, 2008; Accepted February 6, 2009

DOI: 10.3892/or_00000333

\begin{abstract}
To identify the genes and molecular functional pathways involved in esophageal cancer, we analyzed the gene expression profile of esophageal tumor tissue from patients having family history of esophageal cancer by cDNA microarray. Three hundred and fifty differentially expressed genes (26 up-regulated and 324 down-regulated) were identified. Genes involved in humoral immune response (PF4), extracellular matrix organization (COL4A4), metabolism of xenobiotics (EPHX1), TGF- $\beta$ signaling $(S M A D 1)$ and calcium signaling pathways (VDAC1) were down-regulated and genes involved in regulation of actin cytoskeleton (WASL), neuroactive ligand receptor interaction (GRM3), Toll-like receptor (CD14), B-cell receptor (IFITM1) and insulin signaling pathways (FOXO1A) were up-regulated. Validation of differential expression of subset of genes by QRT-PCR and tissue microarray in familial and non-familial cases showed no significant difference in expression of these genes in two groups suggesting familial clustering occurs as result of sharing of common environmental factors. Gene expression profiling of clinical specimens from well characterized populations that have familial clustering of cancer identified molecular mechanism associated with progression of esophageal cancer.
\end{abstract}

\section{Introduction}

Esophageal cancer is among the ten most common malignancies worldwide and ranks as the sixth leading cause of death from cancer (1). Esophageal cancer occurs at very

Correspondence to: Dr Sunita Saxena, Institute of Pathology, Safdarjung Hospital Campus, New Delhi-110029, India

E-mail: sunita_saxena@yahoo.com

Key words: expression profile, familial esophageal cancer, microarray high frequencies in certain parts of China, Iran, South Africa, Uruguay, France and Italy (2). Association of family history with an increased risk of esophageal cancer has been reported in several case control and cohort studies from China, Iran and Japan suggesting possible role of environmental as well as genetic factors $(3,4)$. A high prevalence of esophageal cancer with familial aggregation has also been reported from Assam in the Northeast (NE) region of India with an age-adjusted rate (AAR) of 33/100,000 males $(5,6)$.

Familial clustering of cancer may be due to shared environmental factors or shared genes by family members (7). $\mathrm{Su}$ et al reported that molecular profiles in esophageal squamous cell carcinoma (ESCC) were highly consistent and expression patterns in familial cases were different from those in sporadic cases (3). We have earlier reported that gene expression profile of non-familial ESCC in Assam, a high-risk zone for esophageal cancer in India, are highly consistent with ESCC in China (6).

In the current study, molecular signature of ESCC from high-risk area of India has been studied by gene expression profiling in esophageal cancer patients with family history of esophageal cancer with the aim to elucidate molecular pathogenesis of esophageal cancer in these patients.

\section{Materials and methods}

Selection of patients and collection of samples. Among 317 cases of esophageal cancer registered at Dr Bhubaneshwar Borooah Cancer Institute (BBCI), Guwahati, Assam, 92 (29\%) had family history of esophageal and other cancers besides habit of tobacco and betel quid chewing. Among 92 patients, 45 patients (49\%) had family history of esophageal cancer. Patients diagnosed with metastases and at advanced stage of the disease were excluded from the study. Endoscopic biopsy specimens from tumor and matched normal tissue distant to tumor were collected during diagnostic endoscopy. Part of tumor and normal tissues was preserved in formalin for histopathological diagnosis/ confirmation and remaining tissue was immediately immersed in RNA later solution (Ambion, Austin, TX) and stored at $-70^{\circ} \mathrm{C}$ until processed. Out of 45 patients, 20 
patients with tumor biopsies containing $>80 \%$ tumor were included for gene expression study. Demographic and lifestyle cancer risk factors and clinical data of all 20 patients were collected (Table I). Informed consent was obtained from all the patients to use their surgical specimens and clinicopathological data for the study. Institutional Human Ethics Committee approved this study.

Microarray experiments. Total RNA was isolated from snap-frozen biopsies using the Qiagen (Valencia, CA) RNeasy mini kit and its integrity was examined using the RNA 6000 Nano LabChip on the Agilent 2100 Bioanalyzer (Agilent Technologies, Palo Alto, CA). RNA quantity was determined by the NanoDrop ${ }^{\circledR}$ ND-1000 UV-Vis spectrophotometer (Nanodrop technologies, Rockland, MD). As a reference RNA, we used pool of total RNA from normal esophageal tissue of all patients. Out of 20 patients, RNA samples from nine tumor biopsies with RNA Integrity Number (RIN) of 8 were used for gene expression study by cDNA microarray. Low RNA input fluorescent linear amplification kit (Agilent, Santa Clara, CA) was used for labeling. Individual tumor cRNA was labeled with cyanine 5 and hybridized with cyanine 3-labeled pooled normal esophageal cRNA. The labeled and fragmented cRNAs were hybridized at $65^{\circ} \mathrm{C}$ for $17 \mathrm{~h}$ with human $10 \mathrm{~K}$ cDNA array (University Health Network, Microarray Centre, Toronto, Canada), which contained 9,914 well-characterized human clones.

Microarray image acquisition and data analysis. Hybridized arrays were scanned at $5 \mu \mathrm{m}$ resolution on an Agilent DNA microarray scanner; model G2565AA at $100 \%$ laser power and $30 \%$ PMT at $635 \mathrm{~nm}$ for Cy5-labelled samples and at $532 \mathrm{~nm}$ for Cy3-labeled samples. The resulting TIFF images were analyzed by Agilent Feature Extraction Software 9.1.3, which performed spot localization (Find Spot Algorithm), outlier pixel rejection based on the interquartile range method (Cookie Cutter Algorithm) and flagging of saturated features.

Genespring software version GXV7.3.1 (Agilent Technologies) was used to normalize values for each gene and for further data analysis. Differentially regulated genes were ranked on the basis of signal intensity, normalized ratio, flag value and variance across replicate experiments. Genes were considered to be up-regulated when the median of the normalized ratio was $\geq 2$. Genes were considered to be downregulated when the median of the normalized ratio was $\leq 0.5$. The observed number of differentially expressed genes in each GO category was compared to the corresponding number estimated from a random model (hypergeometric distribution); significance was assessed by a p-value. Hierarchical clustering analysis was also performed with Genespring software GXV 7.3.1. in which the average linkage and Pearson correlation (centered correlation) clustering algorithm was used. The microarray data set was submitted to the GEO repository (GSE 10127) at http://www.ncbi.nlm. nih.gov/geo. Annotations of the bioprocesses, molecular function and cellular localization were obtained using the freely available Gene Ontology [Source database (http://source.stanford.edu) and Biointerpreter software (http://www. genotypic. co.in/biointerpreter)].
Validation of microarray results by quantitative real-time $R T$-PCR analysis. One microgram of tumor and pooled normal RNA was reverse transcribed into cDNA with random primers (High Capacity cDNA archive kit, Applied Biosystems, Foster City, CA). Real-time PCR reactions were performed using an ABI Prism 7000 sequence detection system (Applied Biosystems). Primers and TaqMan probes of five target genes and an internal control gene $18 S$ rRNA were purchased as assays-on-demand from Applied Biosystems (Table II). The thermal cycling conditions included an initial denaturation step at $95^{\circ} \mathrm{C}$ for $10 \mathrm{~min}, 40$ cycles at $95^{\circ} \mathrm{C}$ for $15 \mathrm{sec}$ and $60^{\circ} \mathrm{C}$ for one min. The $2^{-\triangle \Delta C T}$ method was used to calculate relative changes in gene expression determined from real-time quantitative PCR experiments. Validation of microarray results was done in 20 familial ESCC and 10 nonfamilial ESCC cases. Wilcoxon signed rank tests were used to determine the statistical significance of expression difference for each test gene in 20 familial ESCC cases (Table II). The expression profiles of target genes by QRTPCR in two groups (familial and non-familial ESCC cases) were then compared using Mann-Whitney U test. A p-value $\leq 0.05$ was considered significant.

Tissue microarray (TMA)-based immunohistochemical analysis. A TMA was constructed from 120 formalin-fixed, paraffin-embedded blocks of esophageal biopsy samples. These included 20 controls (non-neoplastic esophageal squamous epithelium) and 100-ESCCs, of which 20 biopsies were obtained from familial cases. The tissue cylinders were precisely arrayed into the recipient block with core size of $1.5 \mathrm{~mm}$ using a manual tissue microarrayer (Beecher Instruments, Silver Spring, MD). TMA sections were incubated overnight at $4^{\circ} \mathrm{C}$ with primary antibodies of KRT4 (1:100, Clone 6B10, Novacastra, Newcastle upon Tyne, UK), VEGF (1:50, clone G153-694, BD Biosciences), NF-кB/p65 (1:100, NeoMarkers, Fremont, USA), and anti-collagen IV (1:50, Clone COL-94, Biogenex, USA). The standard streptavidin peroxidase method was employed for immunostaining (8).

\section{Results}

Clinical and epidemiological information of enrolled patients. In $64 \%(59 / 92)$ of cases, cancer occurred in the first-degree relatives whereas in $11 \%(10 / 92)$, cancer occurred in the second-degree relatives. In $\sim 10 \%$ (9/92) of cases, the cancer occurred in both the first-degree and second-degree relatives. The univariate analysis revealed that the risk of developing esophageal cancer was more among subjects whose family history showed occurrence of cancer among the first-degree relatives (OR: 3.1; CI: 1.9-5.3) than the second-degree relatives (OR: 1.3, CI: 0.25-3.2). The estimates also revealed that the risk of developing esophageal cancer was more in subjects whose pee-degree suffered from esophageal cancer (OR: 2.4; CI: 1.1-4.1) than any other cancer (OR: 1.1; CI: $0.32-3.3)$.

Gene expression profiling by cDNA microarray. Four hundred and thirty-eight genes were differentially expressed at least 1-fold in all experiments. The two dimensional hierarchical 
Table I. Demographic and clinical characteristics of esophageal squamous cell carcinoma cases with family history of esophageal cancer.

\begin{tabular}{|c|c|c|c|c|c|c|c|c|c|}
\hline Patient ID & Age & Gender & $\begin{array}{c}\text { Tobacco } \\
\text { chewing habit }\end{array}$ & $\begin{array}{l}\text { Smoking } \\
\text { habit }\end{array}$ & Alcohol use & $\begin{array}{c}\text { Betel } \\
\text { quid use }\end{array}$ & $\begin{array}{l}\text { Family history } \\
\text { of cancer }\end{array}$ & $\begin{array}{c}\text { Pathological } \\
\text { grade }^{\mathrm{a}}\end{array}$ & $\begin{array}{l}\text { Experiments } \\
\text { carried out }^{\mathrm{b}}\end{array}$ \\
\hline EC-116 & 40 & $\mathrm{~F}$ & Yes & No & No & Yes & $\begin{array}{c}\text { Esophageal } \\
\text { cancer (father) }\end{array}$ & $\mathrm{G} 2$ & MA, RT \\
\hline EC-88 & 50 & M & No & Yes & Yes & Yes & $\begin{array}{l}\text { Esophageal } \\
\text { cancer (cousin } \\
\text { brother) }\end{array}$ & G3 & MA, RT \\
\hline EC-99 & 50 & M & No & Yes & No & Yes & $\begin{array}{c}\text { Esophageal } \\
\text { cancer } \\
\text { (mother) }\end{array}$ & $\mathrm{G} 2$ & MA, RT \\
\hline EC-84 & 50 & M & No & Yes & Yes & Yes & $\begin{array}{c}\text { Esophageal } \\
\text { cancer (father) }\end{array}$ & G1 & MA, RT \\
\hline EC-53 & 54 & M & Yes & No & Yes & Yes & $\begin{array}{l}\text { Esophageal } \\
\text { cancer (elder } \\
\text { brother) }\end{array}$ & G3 & MA, RT \\
\hline EC-69 & 70 & M & Yes & Yes & No & Yes & $\begin{array}{l}\text { Esophageal } \\
\text { cancer } \\
\text { (mother) }\end{array}$ & G1 & MA, RT \\
\hline EC-124 & 69 & M & No & Yes & Yes & Yes & $\begin{array}{c}\text { Esophageal } \\
\text { cancer } \\
\text { (brother) }\end{array}$ & $\mathrm{G} 2$ & MA, RT \\
\hline EC-129 & 65 & M & Yes & Yes & No & Yes & $\begin{array}{c}\text { Esophageal } \\
\text { cancer (sister) }\end{array}$ & G1 & MA, RT, \\
\hline EC-83 & 52 & $\mathrm{~F}$ & No & No & No & Yes & $\begin{array}{c}\text { Esophageal } \\
\text { cancer (father) }\end{array}$ & G3 & MA, RT \\
\hline EC-54 & 45 & M & No & Yes & Yes & Yes & $\begin{array}{c}\text { Esophageal } \\
\text { cancer } \\
\text { (mother) }\end{array}$ & G2 & RT \\
\hline EC-72 & 32 & M & No & Yes & No & Yes & $\begin{array}{c}\text { Esophageal } \\
\text { cancer (father) }\end{array}$ & $\mathrm{G} 2$ & RT \\
\hline EC-247 & 45 & $\mathrm{~F}$ & No & No & No & Yes & $\begin{array}{l}\text { Esophageal } \\
\text { cancer } \\
\text { (mother) }\end{array}$ & $\mathrm{G} 2$ & RT \\
\hline EC-248 & 56 & M & Yes & Yes & Yes & Yes & $\begin{array}{c}\text { Esophageal } \\
\text { cancer } \\
\text { (brother) }\end{array}$ & $\mathrm{G} 2$ & RT \\
\hline EC-283 & 55 & $\mathrm{~F}$ & Yes & No & No & Yes & $\begin{array}{c}\text { Esophageal } \\
\text { cancer (father) }\end{array}$ & $\mathrm{G} 2$ & RT \\
\hline EC-291 & 55 & M & No & Yes & No & Yes & $\begin{array}{c}\text { Esophageal } \\
\text { cancer } \\
\text { (brother) }\end{array}$ & G2 & RT \\
\hline EC-65 & 50 & $\mathrm{~F}$ & No & No & No & Yes & $\begin{array}{c}\text { Esophageal } \\
\text { cancer } \\
\text { (parental uncle) }\end{array}$ & G1 & RT \\
\hline EC-217 & 56 & M & Yes & Yes & Yes & Yes & $\begin{array}{l}\text { Esophageal } \\
\text { cancer (elder } \\
\text { brother) }\end{array}$ & G1 & RT \\
\hline EC-244 & 45 & M & Yes & Yes & Yes & Yes & $\begin{array}{c}\text { Esophageal } \\
\text { cancer (elder } \\
\text { brother/father) }\end{array}$ & $\mathrm{G} 2$ & RT \\
\hline
\end{tabular}


Table I. Continued.

\begin{tabular}{ccccccccccc}
\hline Patient ID & Age & Gender & $\begin{array}{c}\text { Tobacco } \\
\text { chewing habit }\end{array}$ & $\begin{array}{c}\text { Smoking } \\
\text { habit }\end{array}$ & Alcohol use & $\begin{array}{c}\text { Betel } \\
\text { quid use }\end{array}$ & $\begin{array}{c}\text { Family history } \\
\text { of cancer }\end{array}$ & $\begin{array}{c}\text { Pathological } \\
\text { grade }^{\mathrm{a}}\end{array}$ & $\begin{array}{c}\text { Experiments } \\
\text { carried out }^{\mathrm{b}}\end{array}$ \\
\hline EC-85 & 85 & M & No & Yes & No & Yes & $\begin{array}{c}\text { Esophageal } \\
\text { cancer (son) }\end{array}$ & G3 & RT \\
EC-187 & 58 & M & Yes & Yes & Yes & Yes & $\begin{array}{l}\text { Esophageal } \\
\text { cancer (father) }\end{array}$ & G2 & RT
\end{tabular}

${ }^{\mathrm{a}} \mathrm{G} 1$, Well differentiated squamous cell carcinoma; G2, moderately differentiated squamous cell carcinoma; G3, poorly differentiated squamous cell carcinoma; ${ }^{\mathrm{b}} \mathrm{MA}$, cDNA microarry; RT, real-time PCR for validation of microarray data.

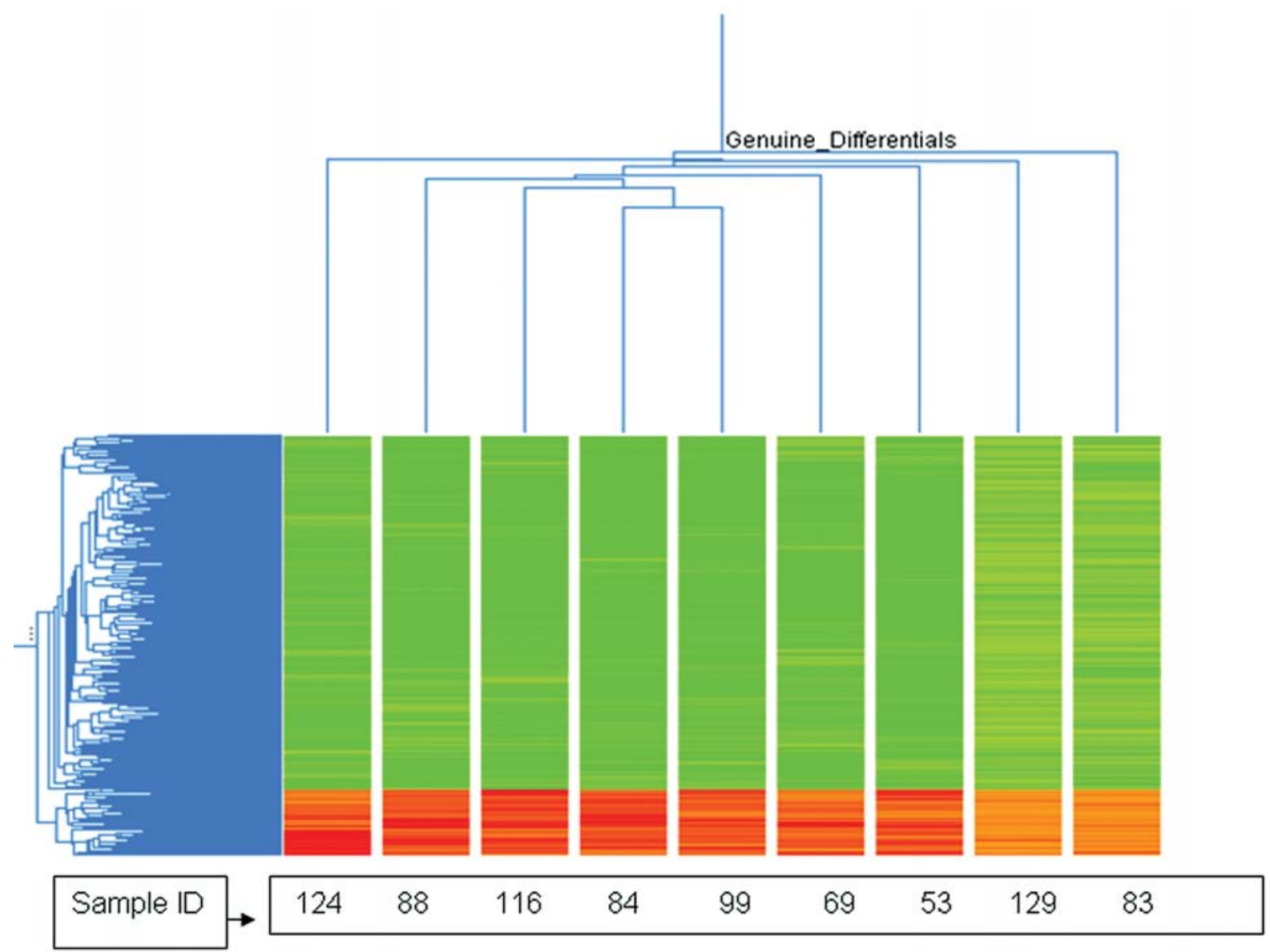

Figure 1. Two-way unsupervised hierarchical clustering (average linkage clustering) of the 438 differentially expressed genes that were over- or underexpressed in tumor vs. normal tissue of nine familial ESCC patients. Red and green colors indicate up-regulated and down-regulated gene expression respectively.

clustering showed that the majority of the differentially expressed genes were significantly down-regulated $(84 \%$, 367 genes), whereas $16 \%$ genes (71 genes) showed upregulation (Fig. 1). Using stringent criteria $(\mathrm{P} \leq 0.05$ and $\geq 1$.2-fold change), 350 differentially expressed genes (26 upregulated and 324 down-regulated) were identified and categorized using the Gene Ontology database into known or probable functional categories on the basis of biological processes and molecular function.

Out of 26 significantly up-regulated genes, genes involved in inflammatory response (CD14), immune response (IFITM1, $V D R, C D 24)$, cell motility (WASL), anti-apoptosis (FOXO1A), glucocorticoid receptor activity $(N R 3 C l)$, steroid hormone receptor activity $(V D R)$, arginase activity $(A R G 1)$ and metabotropic glutamate, GABA-B-like receptor activity (GRM3) were found to be biologically relevant in tumorigenesis (Table IIIA). Out of 324 significantly down-regulated genes, genes involved in extracellular matrix organization (KRT4, COL4A4 and COL14A1), BMP signaling pathway $(S M A D 1)$, epoxide hydrolase activity $(E P H X 1)$, apoptogenic cytochrome c release channel activity (VDAC1,TXNL1), DNA damage response $(S M C 1 A)$, humoral immune response (POU2AF1, PF4, LY9, NFAT5, KLRC1), ion transport $(S L C 22 A 4, S L C 23 A 1)$ and MAP kinase activity (MAPK7, 
Table II. Information on the five genes examined by real-time PCR: location, function, primers and probes.

\begin{tabular}{|c|c|c|c|c|c|c|c|}
\hline Gene & Gene Bank ID & Location & $\begin{array}{l}\text { Gene Expression } \\
\text { status in our study }\end{array}$ & $\begin{array}{l}\text { Putative } \\
\text { function }\end{array}$ & $\mathrm{P}^{\mathrm{a}}$ & Assay ID ${ }^{b}$ & $\begin{array}{l}\text { Amplicon size } \\
\text { (bp) }\end{array}$ \\
\hline CD14 & W56632 & $5 q 22-q 32$ & Up-regulated & Inflammatory response & 0.0002 & Hs02621496_s1 & 140 \\
\hline$A R G 1$ & AA149501 & $6 q 23$ & Up-regulated & Arginine catabolism & 0.0002 & Hs00163660_m1 & 86 \\
\hline PF4 & AA024929 & $4 q 12-q 21$ & Down-regulated & $\begin{array}{l}\text { Negative regulation } \\
\text { of Angiogenesis }\end{array}$ & 0.0028 & Hs00236998_m1 & 86 \\
\hline EPHXI & AA838691 & $1 q 42.1$ & Down-regulated & Xenobiotic metabolism & 0.0002 & Hs01116802_m1 & 89 \\
\hline MAPK7 & H39192 & $17 \mathrm{p} 11.2$ & Down-regulated & MAP kinase activity & 0.0012 & Hs00964720_g1 & 97 \\
\hline
\end{tabular}

${ }^{a}$ Wilcoxon signed rank tests were used to determine the statistical significance of expression difference for each test gene in 20 samples. Statistical significance was defined as $\mathrm{P}<0.05$. ${ }^{\mathrm{b}} \mathrm{m} 1$ denotes that assay's probe spans an exon junction and will not detect genomic DNA. s1 denotes that assay's primers and probes are designed within a single exon and will detect genomic DNA. g denotes that assay may detect genomic DNA.

\section{A}

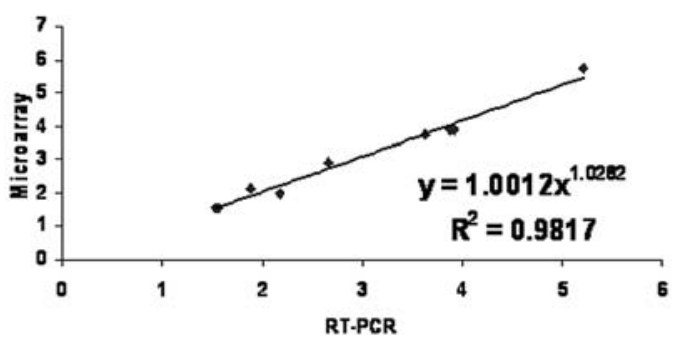

C

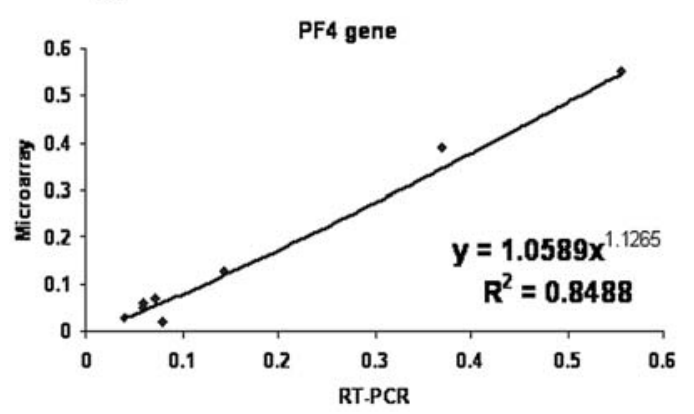

$\mathrm{E}$

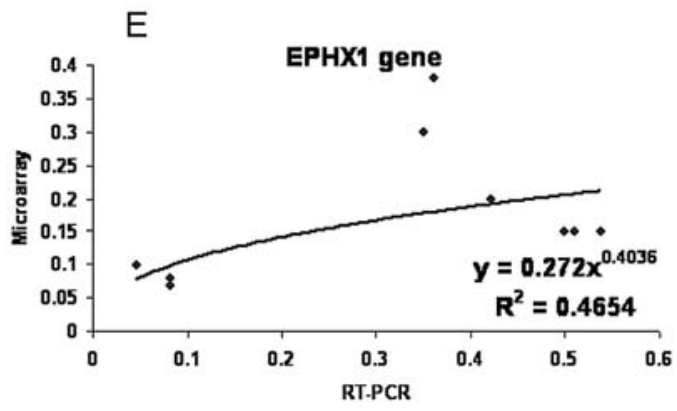

SHC1) were selected on the basis of biological relevance in tumorigenesis (Table IIIB).

Validation of microarray results with quantitative real-time $P C R$. Five top ranked genes viz ARG1, CD14, PF4, MAPK7
B

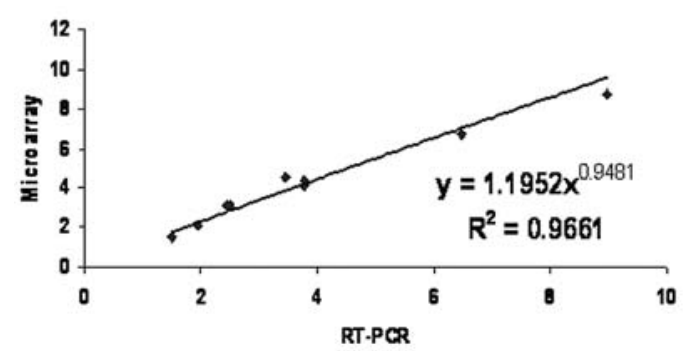

D

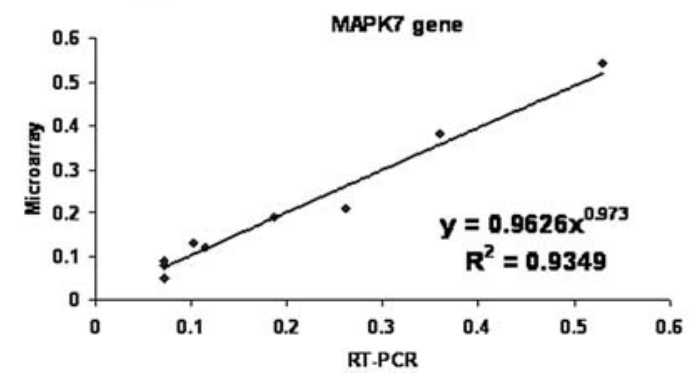

Figure 2. Regression plots for fold change by microarray (Y-axis) and quantitative real-time PCR assay (X-axis) for ARG1 (A), CD14 (B), PF4 (C), MAPK7 (D) and EPHX1 (E).

and $E P H X 1$ were selected to confirm the microarray results with real-time RT-PCR. Regression plot analyses for the five genes showed positive correlation between the gene expression measured by cDNA microarray and real-time RT-PCR (Fig. 2). Pearson correlation coefficient of each gene was ARG1 0.99, CD14 0.98, PF4 0.99, MAPK7 0.98 and EPHX1 0.414. Quantitative real-time PCR performed on all the nine patient specimens previously arrayed, 11 other familial ESCC 

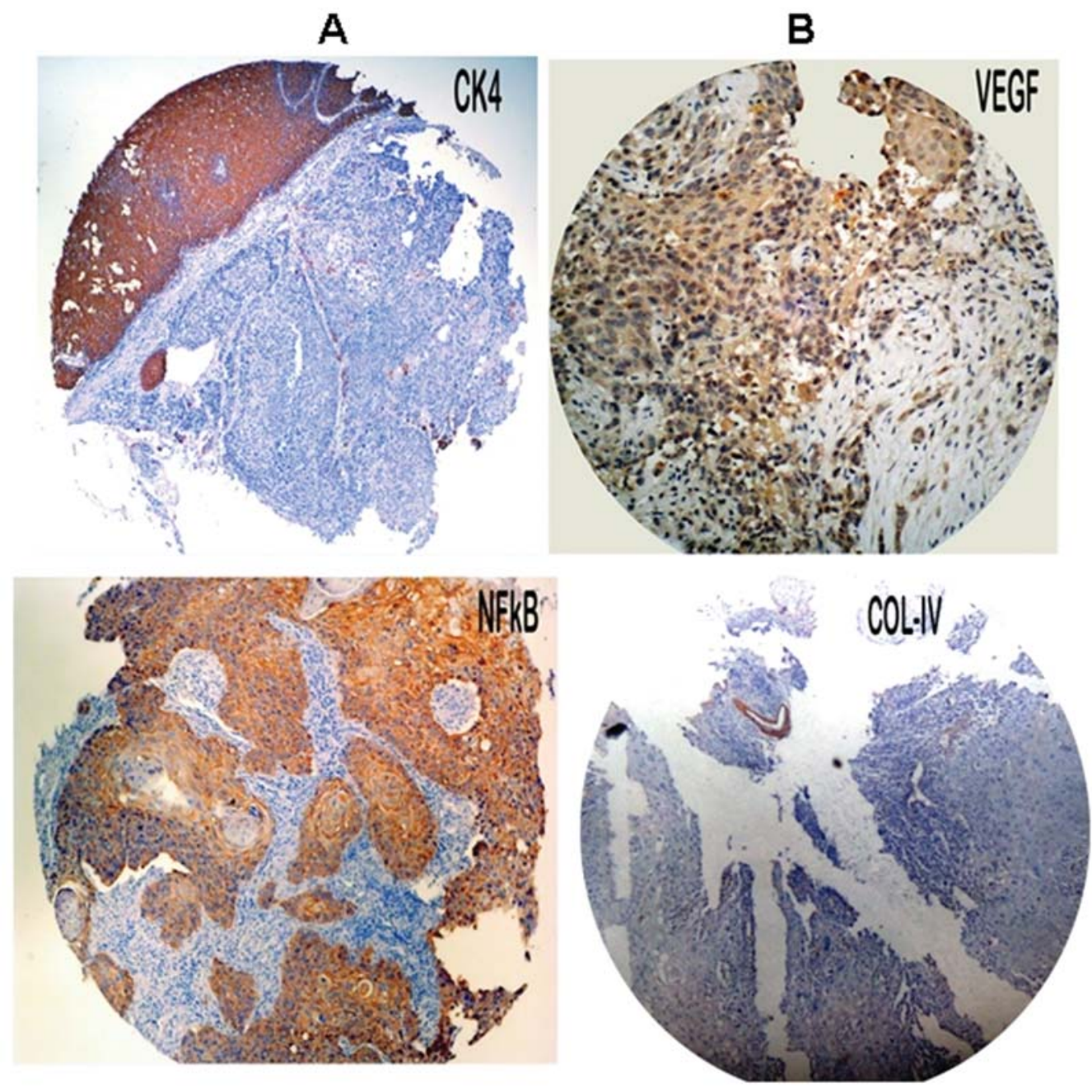

C

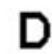

Figure 3. Photomicrograph of tissue microarray cores from esophageal tumor biopsies obtained from familial ESCC patients showing negative immunostaining for KRT4 (A) and Collagen IV (D) and positive immunostaining for VEGF (B) and NF-kB (C).

patient specimens and 10 non-familial ESCC, indicated that $A R G 1$ and $C D 14$ were consistently up-regulated and $P F 4$, $M A P K 7$ and EPHX1 were consistently down-regulated in all patient specimens (Table II). No statistical significant difference $(\mathrm{P}=0.1508-0.5358)$ was observed in relative gene expression for five target genes in between familial and non-familial ESCC groups.

TMA-based immunohistochemical analysis. Differential expression of KRT4 and COL4 genes identified in cDNA microarray analysis were validated at protein level using immunohistochemistry on TMA. We also studied expression of $V E G F$ (which is downstream target gene of PF4 and $M A P K 7$ ) and $N F-\kappa B$ (which is down-stream target gene of $C D 14$ ) at protein level. The cytoplasmic staining was considered positive for CK4 (KRT4), VEGF, NF- $\mathrm{KB}$ and collagen 4 (COL4) and was scored as $<5 \%$ or no staining $=0$, $5-25 \%=1,26-50 \%=2,51-100 \%=3$. Expression of KRT4 was found only in overlying non-neoplastic epithelium and absent in tumor cells (14 out of 20 showing a score of 0 while remaining 6 score of 1). VEGF showed expression in tumor cells with score of 2 in $8(40 \%)$ cases and with score of 3 in $12(60 \%)$ cases. NF- $\mathrm{kB}$ showed a diffuse and strong expression in tumor cells with score of 3 in all familial cases (100\%). The COL4A4 was not expressed in the tumor cells. The staining intensity was not graded as all the cores were more or less uniformly stained (Fig. 3). Staining pattern in tumor cores from non-familial ESCC cases showed similar staining pattern as in familial cases.

\section{Discussion}

To the best of our knowledge, this is the first study that gives an insight into the genes and molecular pathways that may be playing an important role in the familial aggregation of esophageal cancer in high-risk area of India. According to Hanahan and Weinberg (9), tumorigenesis requires six essential alterations to normal cell physiology: self-sufficiency in growth signals, insensitivity to growth inhibition, evasion of apoptosis, immortalization, sustained angiogenesis and tissue invasion and metastasis. In addition, another component of cancer progression is the failure of the host immune response 
Table III. Biologically relevant and statistically significant up-regulated and down-regulated genes in esophageal cancer patients with family history of esophageal cancer.

\begin{tabular}{|c|c|c|c|c|c|c|c|}
\hline Genes & Gene symbol & Gene bank ID & $\begin{array}{l}\text { Chromosomal } \\
\text { location }\end{array}$ & $\begin{array}{l}\text { Median fold } \\
\text { change }\end{array}$ & GO category & P-value & Pathway ${ }^{b}$ \\
\hline CD14 & $C D 14$ & W56632 & $5 q 22-q 32$ & 2.05 & $\begin{array}{l}\text { GO: } 6954: \\
\text { inflammatory } \\
\text { response }\end{array}$ & 0.00 & $\begin{array}{l}\text { Toll-like } \\
\text { receptor } \\
\text { signaling }\end{array}$ \\
\hline Arginase & $A R G 1$ & AA149501 & $6 q 23$ & 1.55 & $\begin{array}{l}\text { GO: } 4053 \text { : arginase } \\
\text { activity }\end{array}$ & 0.01 & $\begin{array}{l}\text { Urea cycle } \\
\text { and } \\
\text { metabolism } \\
\text { of amino } \\
\text { groups }\end{array}$ \\
\hline $\begin{array}{l}\text { Glutamate } \\
\text { receptor, } \\
\text { metabotropic } 3\end{array}$ & GRM3 & N98673 & 7q21.1-q21.2 & 1.54 & $\begin{array}{l}\text { GO: } 8067: \\
\text { metabotropic glutamate, } \\
\text { GABA-B-like } \\
\text { receptor activity }\end{array}$ & 0.04 & $\begin{array}{l}\text { Neuroactive } \\
\text { ligand } \\
\text { receptor } \\
\text { interaction }\end{array}$ \\
\hline $\begin{array}{l}\text { Forkhead } \\
\text { box O1A } \\
\text { (rhabdomy- } \\
\text { osarcoma) }\end{array}$ & FOXO1A & W32908 & $13 q 14.1$ & 1.43 & $\begin{array}{l}\text { GO: 6916: anti- } \\
\text { apoptosis }\end{array}$ & 0.03 & $\begin{array}{l}\text { Insulin } \\
\text { signaling }\end{array}$ \\
\hline $\begin{array}{l}\text { Interferon- } \\
\text { induced } \\
\text { trans- } \\
\text { membrane } \\
\text { protein } 1 \\
(9-27)\end{array}$ & IFITMI & H49853 & $11 \mathrm{p} 15.5$ & 1.41 & $\begin{array}{l}\text { GO: 6955: immune } \\
\text { response }\end{array}$ & 0.00 & $\begin{array}{l}\text { B-cell } \\
\text { receptor } \\
\text { signaling }\end{array}$ \\
\hline $\begin{array}{l}\text { Wiskott- } \\
\text { Aldrich } \\
\text { syndrome- } \\
\text { like }\end{array}$ & $W A S L$ & AI271884 & $7 q 31.3$ & 1.41 & $\begin{array}{l}\text { GO: } 6928: \text { cell } \\
\text { motility }\end{array}$ & 0.01 & $\begin{array}{l}\text { Regulation } \\
\text { of actin } \\
\text { cytoskeleton }\end{array}$ \\
\hline $\begin{array}{l}\text { Nuclear } \\
\text { receptor } \\
\text { subfamily 3, } \\
\text { group C, } \\
\text { member 1 } \\
\text { glucocorticoid } \\
\text { receptor }\end{array}$ & $N R 3 C 1$ & AA053901 & $5 q 31.3$ & 1.32 & $\begin{array}{l}\text { GO: } 4883 \text { : } \\
\text { glucocorticoid } \\
\text { receptor activity }\end{array}$ & 0.00 & $\begin{array}{l}\text { Neuroactive } \\
\text { ligand } \\
\text { receptor } \\
\text { interaction }\end{array}$ \\
\hline $\begin{array}{l}\text { Vitamin D } \\
(1,25-\text { dihy- } \\
\text { droxyvitamin } \\
\text { D3) receptor }\end{array}$ & $V D R$ & BG149860 & $12 \mathrm{q} 13.11$ & 1.20 & $\begin{array}{l}\text { GO: } 3707 \text { : steroid } \\
\text { hormone receptor } \\
\text { activity }\end{array}$ & 0.00 & $\mathrm{NA}^{\mathrm{c}}$ \\
\hline $\begin{array}{l}\text { Gardner- } \\
\text { Rasheed } \\
\text { feline sarcoma } \\
\text { viral (v-fgr) } \\
\text { oncogene } \\
\text { homolog }\end{array}$ & $F G R$ & W81591 & 1p36.2-p36.1 & 1.42 & GO: 6928: cell motility & 0.00 & $\begin{array}{l}\text { Focal } \\
\text { adhesion }\end{array}$ \\
\hline
\end{tabular}

B, Down-regulated genes

\begin{tabular}{lllllll}
\hline Keratin 4 & KRT4 & AA629189 $12 \mathrm{q} 12-\mathrm{q} 13$ & -5.20 & $\begin{array}{l}\text { GO: 30198: extra- } \\
\text { cellular matrix organ- } \\
\text { ization and biogenesis }\end{array}$ & $\begin{array}{l}\text { Cell } \\
\text { communication }\end{array}$
\end{tabular}


Table IIIB. Continued.

\begin{tabular}{|c|c|c|c|c|c|c|c|}
\hline Genes & Gene symbol & Gene bank ID & $\begin{array}{l}\text { Chromosomal } \\
\text { location }\end{array}$ & $\begin{array}{l}\text { Median fold } \\
\text { change }\end{array}$ & GO category & P-value & Pathway ${ }^{b}$ \\
\hline $\begin{array}{l}\text { SMAD, } \\
\text { mothers } \\
\text { against DPP } \\
\text { homolog } 1 \\
(\text { Drosophila) }\end{array}$ & $S M A D 1$ & R83757 & $4 q 31$ & -2.48 & $\begin{array}{l}\text { GO: } 30509: \text { BMP } \\
\text { signaling pathway }\end{array}$ & 0.00 & $\begin{array}{l}\text { TGF- } 3 \\
\text { signaling }\end{array}$ \\
\hline $\begin{array}{l}\text { Epoxide } \\
\text { hydrolase } 1, \\
\text { microsomal } \\
\text { (xenobiotic) }\end{array}$ & $E P H X 1$ & AA838691 & $1 q 42.1$ & -2.76 & $\begin{array}{l}\text { GO: 4301: epoxide } \\
\text { hydrolase activity }\end{array}$ & 0.01 & $\begin{array}{l}\text { Metabolism of } \\
\text { xenobiotics by } \\
\text { cytochrome } \\
\text { p450 }\end{array}$ \\
\hline $\begin{array}{l}\text { Voltage- } \\
\text { dependent } \\
\text { anion } \\
\text { channel } 1\end{array}$ & $V D A C 1$ & AA025089 & $5 q 31$ & -2.31 & $\begin{array}{l}\text { GO: } 15283: \\
\text { apoptogenic } \\
\text { cytochrome c } \\
\text { release channel } \\
\text { activity }\end{array}$ & 0.00 & $\begin{array}{l}\text { Calcium } \\
\text { signaling }\end{array}$ \\
\hline $\begin{array}{l}\text { Platelet } \\
\text { factor } 4 \\
\text { [chemokine } \\
\text { (C-X-C) } \\
\text { motif] ligand } 4\end{array}$ & PF4 & AA024929 & $4 q 12-q 21$ & -4.11 & $\begin{array}{l}\text { GO: 6959: humoral } \\
\text { immune } \\
\text { response }\end{array}$ & 0.00 & $\begin{array}{l}\text { Leukocyte } \\
\text { transendo- } \\
\text { thelial } \\
\text { migration }\end{array}$ \\
\hline $\begin{array}{l}\text { Solute carrier } \\
\text { family } 22 \\
\text { (organic } \\
\text { cation } \\
\text { transporter), } \\
\text { member } 4\end{array}$ & SLC22A4 & N26836 & $5 q 31.1$ & -2.94 & $\begin{array}{l}\text { GO: } 6811: \text { ion } \\
\text { transport }\end{array}$ & 0.00 & $\mathrm{NA}^{\mathrm{c}}$ \\
\hline $\begin{array}{l}\text { Mitogen } \\
\text { activated } \\
\text { protein } \\
\text { kinase } 7\end{array}$ & MAPK7 & H39192 & $17 \mathrm{p} 11.2$ & -2.89 & $\begin{array}{l}\text { GO: } 4707: \\
\text { MAP kinase } \\
\text { activity }\end{array}$ & 0.00 & $\begin{array}{l}\text { GnRH } \\
\text { signaling/gap } \\
\text { junction }\end{array}$ \\
\hline $\begin{array}{l}\text { Killer cell } \\
\text { lectin-like } \\
\text { receptor } \\
\text { subfamily C, } \\
\text { member } 1\end{array}$ & KLRC1 & AA913480 & $12 \mathrm{p} 13$ & -2.39 & $\begin{array}{l}\text { GO: } 6959 \text { : } \\
\text { humoral immune } \\
\text { response }\end{array}$ & 0.00 & $\begin{array}{l}\text { Natural killer } \\
\text { cell-mediated } \\
\text { cytotoxicity }\end{array}$ \\
\hline $\begin{array}{l}\text { Nuclear factor } \\
\text { of activated } \\
\text { T-cells } 5\end{array}$ & NFAT5 & H60999 & $16 \mathrm{q} 22.1$ & -2.23 & $\begin{array}{l}\text { GO: } 6959 \text { : } \\
\text { humoral immune } \\
\text { response }\end{array}$ & 0.00 & $\begin{array}{l}\text { Natural killer } \\
\text { cell-mediated } \\
\text { cytotoxicity }\end{array}$ \\
\hline $\begin{array}{l}\text { Collagen, } \\
\text { type XIV, } \\
\alpha 1 \text { (undulin) }\end{array}$ & COL14A1 & AA167222 & $8 q 23$ & -2.01 & $\begin{array}{l}\text { GO: } 30198 \text { : } \\
\text { extracellular matrix } \\
\text { organization and } \\
\text { biogenesis }\end{array}$ & 0.00 & $\begin{array}{l}\text { Cell } \\
\text { communication }\end{array}$ \\
\hline $\begin{array}{l}\text { Collagen, } \\
\text { type IV }, \alpha 4\end{array}$ & COLAA4 & H67349 & $2 q 35-q 37$ & -2.18 & $\begin{array}{l}\text { GO: } 30198 \text { : } \\
\text { extracellular matrix } \\
\text { organization and } \\
\text { biogenesis }\end{array}$ & 0.00 & $\begin{array}{l}\text { Cell } \\
\text { communication }\end{array}$ \\
\hline $\begin{array}{l}\text { SHC } \\
\text { (Src } \\
\text { homology } 2 \\
\text { domain containi } \\
\text { transforming } \\
\text { protein } 1\end{array}$ & SHCl & R52961 & $1 \mathrm{q} 21$ & -3.28 & $\begin{array}{l}\text { GO: } 4707: \text { MAP } \\
\text { kinase activity }\end{array}$ & 0.00 & $\begin{array}{l}\text { Natural killer } \\
\text { cell-mediated } \\
\text { cytotoxicity }\end{array}$ \\
\hline
\end{tabular}


Table IIIB. Continued.

\begin{tabular}{|c|c|c|c|c|c|c|c|}
\hline Genes & Gene symbol & Gene bank ID & $\begin{array}{l}\text { Chromosomal } \\
\text { location }\end{array}$ & $\begin{array}{l}\text { Median fold } \\
\text { change }\end{array}$ & GO category & P-value ${ }^{a}$ & Pathway ${ }^{b}$ \\
\hline $\begin{array}{l}\text { Solute } \\
\text { carrier } \\
\text { family } 23\end{array}$ & $S L C 23 A 1$ & AI334656 & $5 q 31.2-q 31.3$ & -3.03 & $\begin{array}{l}\text { GO: } 6811: \text { ion } \\
\text { transport }\end{array}$ & 0.00 & $\mathrm{NA}^{\mathrm{c}}$ \\
\hline $\begin{array}{l}\text { Lymphocyte } \\
\text { antigen } 9\end{array}$ & $L Y 9$ & AI056539 & 1q21.3-q22 & -2.43 & $\begin{array}{l}\text { GO: 6959: humoral } \\
\text { immune response }\end{array}$ & 0.00 & $\mathrm{NA}^{\mathrm{c}}$ \\
\hline $\begin{array}{l}\text { POU domain, } \\
\text { class } 2, \\
\text { associating } \\
\text { factor } 1\end{array}$ & POU2AF1 & AI028546 & $11 q 23.1$ & -2.36 & $\begin{array}{l}\text { GO: 6959: humoral } \\
\text { immune response }\end{array}$ & 0.00 & $\mathrm{NA}^{\mathrm{c}}$ \\
\hline $\begin{array}{l}\text { SMC1 } \\
\text { structural } \\
\text { maintenance of } \\
\text { chromosomes } \\
\text { 1-like 1 (yeast) }\end{array}$ & SMC1A & AA598887 & Xp11.22-p11.21 & -2.43 & $\begin{array}{l}\text { GO: 42770: DNA } \\
\text { damage response }\end{array}$ & 0.01 & Cell cycle \\
\hline $\begin{array}{l}\text { Thioredoxin- } \\
\text { like } 1\end{array}$ & TXNL1 & AA078976 & $18 q 21.31$ & -2.19 & $\begin{array}{l}\text { GO: } 15283: \\
\text { apoptogenic } \\
\text { cytochrome c } \\
\text { release channel } \\
\text { activity }\end{array}$ & 0.00 & $\mathrm{NA}^{\mathrm{c}}$ \\
\hline
\end{tabular}

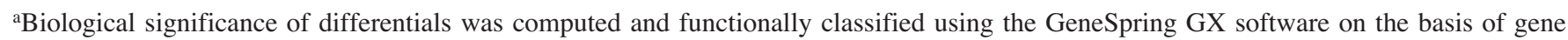
ontology. GeneSpring GX software depicts the biologically significant ontology for any given gene list as follows: number of genes in the array known to be present in the ontology category vs. number of genes in the array that are differentially regulated. bPathways were obtained using enrichment analysis based on gene ontology categories using the Biointerpreter software and DAVID knowledgebase (http://david.abcc.ncifcrf.gov/knowledgebase). ${ }^{\mathrm{N}} \mathrm{NA}$, No information available.

to recognize tumor cells $(10,11)$. Molecular profiling of esophageal cancer with familial clustering showed deregulation of most of these physiological mechanisms in the current study (Fig. 4). On the basis of functional annotation, genes responsible for inflammatory response, immune response, angiogenesis, cell migration and cell proliferation were found significantly de-regulated in these cases.

Genes (KRT4, COL4A4) involved in extracellular matrix organization and cell communication pathway showed significant down-regulation in present study. $C K 4$ influences the formation of cytoskeletal cells and its low expression has been reported earlier in upper aero-digestive tract tumors (8). Loss of expression of type IV collagen $\alpha 5$ and $\alpha 6$ chains, associated with the hypermethylation of their promoter region, has also been reported in colorectal cancer (12).

Platelet factor 4 ( $P F-4)$-a CXC-chemokine-involved in humoral immune response, leukocyte transendothelial migration pathway and inflammatory processes, showed significant down-regulation in our study. PF4 inhibits T cell function by down-modulating cell proliferation and cytokine release. In addition, $\mathrm{PF}-4$ has strong anti-angiogenic properties that inhibit endothelial cell proliferation and migration, in vitro and in vivo angiogenesis, tumor-associated neovascularization and tumor growth (13). PF-4 inhibits VEGF-induced mitogenactivated protein kinase (MAPK) signaling pathways comprising Raf1, MEK1/2 and ERK1/2 genes (14). MAPK7 (ERK5) was found to be down-regulated in our study. MAPK7 deficiency leads to an increased expression of VEGF, deregulation of which has been shown to impede angiogenic remodeling and vascular stabilization. Increased VEGF expression in a hypoxic environment promotes vessel growth, angiogenesis and tumor growth (15).

Microsomal epoxide hydrolase 1 (EPHX1), involved in metabolism of xenobiotics by CYP450, plays an important role in both the activation and detoxification of tobaccoderived carcinogens (16). In addition, SLC22A4, a novel proton antiporter gene that plays a role in the renal excretion of xenobiotics and their metabolites also showed downregulation in our study. Voltage-dependent anion channel $(V D A C 1)$ gene, which is involved in calcium signaling and apoptosis inducing pathway, showed down-regulation in present study. VDACl controls pro- and anti-apoptotic Bcl2-family proteins by regulating the release of cytochrome $\mathrm{c}$ and apoptotic proteins in the inter-membrane space (17). $S M A D 1$ that is involved in TGF- $\beta$ or BMP (bone morphogenetic proteins) signaling pathway and helps in tumor progression, showed significant down-regulation in the present study. BMPs are involved in wide range of biological activities including cell growth, apoptosis, morphogenesis, development and immune responses. An earlier study has reported that 


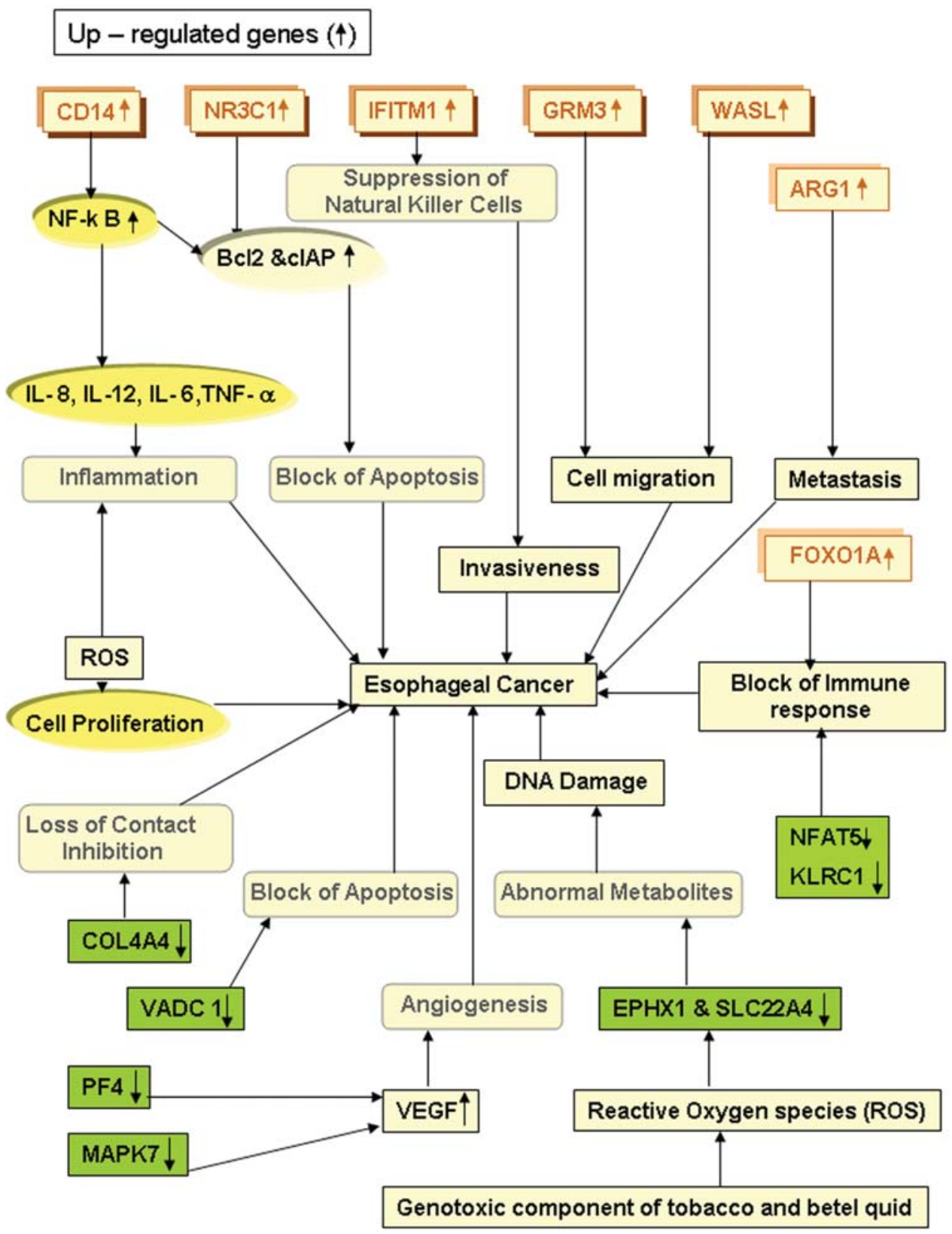

Down - regulated genes $(\downarrow)$

Figure 4. Schematic illustration of differentially expressed genes involved in molecular mechanism of esophageal tumorigenesis. $\uparrow$ Indicates up-regulated genes and $\downarrow$ indicates down-regulated genes. The key differentially expressed genes $(C D 14, A R G 1, E P H X 1, M A P K 7, P F 4, C O L 4 A 4$ and $C K 4)$ were validated by RT-PCR/tissue microarray.

SMAD1 signaling is low in androgen-regulated growth of prostate cancer, is activated after castration and again decreases in hormone-independent tumors (18).

CD14, which is involved in Toll-like receptor (TLR) signaling pathway and inflammatory response, showed significant up-regulation in our study. CD14 induces inflammatory response via MyD88, TIRAP and TRAF6 leading to NF- $\mathrm{KB}$ activation and cytokine secretion. The activation of TLR signaling in tumor cells induces the synthesis of proinflammatory factors and immunosuppressive molecules, which enhance the resistance of tumor cells to cytotoxic lymphocyte attack and lead to immune evasion (19).
WASL gene, which is involved in cell motility and regulation of actin cytoskeleton, showed up-regulation in present study. Bourguignon et al (20) earlier reported that $N W A S P$ (WASL) played a pivotal role in regulating CD44-ErbB2 interaction, ss-catenin signaling and actin cytoskeleton functions that were required for tumor-specific behavior such as transcriptional up-regulation and tumor cell migration. Overexpression of interferon inducible 9-27 (IFITM1) gene, which is involved in B-cell receptor signaling pathway and immune response, has been reported to play a role in malignant progression by suppressing natural killer cells and by increasing the invasive potential of gastric cancer cells (21). 
Gene involved in arginine metabolism ( $A R G l)$ has been found to be up-regulated in these ESCC patients. Myeloid suppressor cells (MSCs) producing high levels of arginase I block T cell function by depleting l-arginine in cancer, chronic infections, and trauma patients. In cancer, infiltration of MSCs in circulation is an important mechanism for tumor evasion and impairs the therapeutic potential of cancer immunotherapies (22). Overexpression of arginase in colorectal carcinoma is associated with metastasis (23).

FOXO1A, a transcription factor that is involved in antiapoptosis and insulin signaling pathway, showed up-regulation in our study. It can promote tumor growth and tissue invasion while inhibiting local inflammatory and immune responses. FOXO1A is the pathogenetic marker for alveolar rhabdomyosarcoma, an aggressive form of childhood cancer (24). Constitutive phosphorylation of the FOXO1A transcription factor has also been reported as a prognostic variable in gastric cancer (25).

NR3C1 (the glucocorticoid receptor family) and GRM3 (GABA-B-like receptor activity) genes, showing up-regulation in present study, are associated with enhanced anti-apoptotic effect and tumor cell migration, respectively. Both are involved in the neuroactive ligand receptor interaction pathway. The ligand-activated glucocorticoid receptor activates the anti-apoptotic $\mathrm{Bcl}-2$ family protein $\mathrm{Bcl}-\mathrm{x}(\mathrm{L})$ that inhibits apoptosis and caspase- 3 activity in fibrosarcoma cells (26). Activation of the glucocorticoid receptor in epithelial ovarian cancer cells has earlier been reported to have an enhanced cellular expression level of cIAP2 and antiapoptotic effect (27). The multifunctional G-protein-coupled metabotropic glutamate receptor (mGluR) family contributes to tumor cell migration and invasion in oral cancer (28).

Several differentially regulated genes in familial ESCC are functionally annotated to immune response category. Up-regulation of CD14, WASL, IFITM1, FOXO1A, GRM3, $A R G 1$ and NR3Cl genes is found to be associated with suppression of NK cells, inhibition of immune response, immune evasion, tumor cell migration, invasion, metastasis and anti-apoptosis, respectively. Down-regulation of $P F 4$, SMAD1, SLC22A4, MAPK7, KLRC1, NFAT5, SHC1, LY9, $P O U 2 A F 1$ and VDAC1 genes may be involved in invasion, inhibition of humoral immune response, angiogenesis and anti-apoptosis respectively in these familial ESCC cases. Furthermore, the data presented here will not only provide important information on tumorigenesis of this tumor, but also facilitate the identification of candidate genes that could be used as therapeutic targets for the treatment of patients with this tumor.

Validation of $C D 14, A R G 1, P F 4, M A P K 7$ and $E P H X 1$ genes at the mRNA level by real-time PCR and KRT4, COL4, $N F-\kappa B$ and $V E G F$ genes at the protein level by tissue microarray did not show any difference in familial and nonfamilial ESCC cases from the same high-risk area of India, suggesting that familial clustering of cancer in these patients is more due to shared environmental factors rather than shared genes by family members.

In this study, the use of high throughput genomic technology in clinical specimens from well characterized populations that have familial clustering of cancer may lead to identification of molecular mechanism associated with progression of esophageal cancer. Functional analyses of these genes will lead to better understanding of the development and progression of ESCC.

\section{Acknowledgements}

The authors are thankful to Jagannath Sharma of BBCI for histopathological analysis, Manisha Sangma, L.C. Singh and Shweta Agarwal of Institute of Pathology, for immunohistochemistry and real-time PCR.

\section{References}

1. Parkin DM, Bray F, Ferlay J and Pisani P: Global cancer statistics, 2002. CA Cancer J Clin 55: 74-108, 2005.

2. Stoner GD and Gupta A: Etiology and chemoprevention of esophageal squamous cell carcinoma. Carcinogenesis 22: 1737-1746, 2001

3. Su H, Hu N, Shih J, Hu Y, Wang QH, Chuang EY, Roth MJ, Wang C, Goldstein AM, Ding T, Dawsey SM, Giffen C, Emmert-Buck MR and Taylor PR: Gene expression analysis of esophageal squamous cell carcinoma reveals consistent molecular profiles related to a family history of upper gastrointestinal cancer. Cancer Res 63: 3872-3876, 2003

4. Nyren O and Adami H-O: Esophageal Cancer. In: Text Book of Cancer Epidemiology, H-O Adami, D Hunter, D Trichopoulos (eds). Oxford University Press, pp137-155, 2002.

5. Phukan RK, Mahanta J and Hazarika NC: Annual Report, Regional Medical Research Center, Dibrugarh, Assam, India. pp21-24: 2005-2006.

6. Chattopadhyay I, Kapur S, Purkayastha J, Phukan R, Kataki A Mahanta J and Saxena S: Gene expression profile of esophageal cancer in North East India by cDNA microarray analysis. World J Gastroenterol 13: 1438-1444, 2007.

7. Hemminki $\mathrm{K}$ and Li X: Familial risks of cancer as a guide to gene identification and mode of inheritance. Int J Cancer 110: 291-294, 2004.

8. Xue LY, Hu N, Song YM, Zou SM, Shou JZ, Qian LX, Ren LQ, Lin DM, Tong T, He ZG, Zhan QM, Taylor PR and Lu N: Tissue microarray analysis reveals a tight correlation between protein expression pattern and progression of esophageal squamous cell carcinoma. BMC Cancer: 6296, 2006.

9. Hanahan D and Weinberg RA: The hallmarks of cancer. Cell 100: 57-70, 2000.

10. Perwez HS and Curtis CH: Inflammation and cancer: An ancient link with novel potentials. Int J Cancer 121: 2373-2380, 2007.

11. Federico A, Morgillo F, Tuccillo C, Ciardiello F and Loguercio C: Chronic inflammation and oxidative stress in human carcinogenesis. Int J Cancer 121: 2381-2386, 2007.

12. Ikeda K, Iyama K, Ishikawa N, Egami H, Nakao M, Sado Y, Ninomiya $Y$ and Baba H: Loss of expression of type IV collagen alpha5 and alpha6 chains in colorectal cancer associated with the hypermethylation of their promoter region. Am J Pathol 168: 856-865, 2006

13. Yamaguchi K, Ogawa K, Katsube T, Shimao K, Konno S, Shimakawa T, Yoshimatsu K, Naritaka Y, Yagawa H and Hirose K: Platelet factor 4-gene transfection into tumor cells inhibits angiogenesis, tumor growth and metastasis. Anticancer Res 25: 847-851, 2005

14. Sulpice E, Contreres JO, Lacour J, Bryckaert M and Tobelem G: Platelet factor 4 disrupts the intracellular signalling cascade induced by vascular endothelial growth factor by both KDR dependent and independent mechanisms. Eur J Biochem 271: 3310-3318, 2004

15. Sohn SJ, Sarvis BK, Cado D and Winoto A: ERK5 MAPK regulates embryonic angiogenesis and acts as a hypoxia-sensitive repressor of vascular endothelial growth factor expression. J Biol Chem 277: 43344-43351, 2002

16. Kiyohara C, Yoshimasu K, Takayama K and Nakanishi Y: EPHX1 polymorphisms and the risk of lung cancer: a HuGE review. Epidemiology 17: 89-99, 2006.

17. Shoshan-Barmatz V, Israelson A, Brdiczka D and Sheu SS: The voltage-dependent anion channel (VDAC): function in intracellular signalling, cell life and cell death. Curr Pharm Des 12: $2249-2270,2006$ 
18. Qiu T, Grizzle WE, Oelschlager DK, Shen X and Cao X: Control of prostate cell growth: BMP antagonizes androgen mitogenic activity with incorporation of MAPK signals in Smad1. EMBO J 26: 346-357, 2007.

19. Huang B, Zhao J, Unkeless JC, Feng ZH and Xiong H: TLR signaling by tumor and immune cells: a double-edged sword. Oncogene 27: 218-224, 2008.

20. Bourguignon LY, Peyrollier K, Gilad E and Brightman A: Hyaluronan-CD44 interaction with neural Wiskott-Aldrich syndrome protein (N-WASP) promotes actin polymerization and ErbB2 activation leading to beta-catenin nuclear translocation, transcriptional up-regulation, and cell migration in ovarian tumor cells. J Biol Chem 282: 1265-1280, 2007.

21. Yang Y, Lee JH and Kim KY: The interferon-inducible 9-27 gene modulates the susceptibility to natural killer cells and the invasiveness of gastric cancer cells. Cancer Lett 221: 191-200, 2005

22. Rodriguez PC, Hernandez CP and Quiceno D: Arginase I in myeloid suppressor cells is induced by COX-2 in lung carcinoma. J Exp Med 202: 931-939, 2005.

23. Mielczarek M, Chrzanowska A, Scibior D, Skwarek A, Ashamiss F, Lewandowska K and Baranczyk-Kuzma A: Arginase as a useful factor for the diagnosis of colorectal cancer liver metastases. Int J Biol Markers 21: 40-44, 2006.
24. Nabarro S, Himoudi N and Papanastasiou A: Coordinated oncogenic transformation and inhibition of host immune responses by the PAX3-FKHR fusion oncoprotein. J Exp Med 202: 1399-1410, 2005

25. Kim JH, Kim MK and Lee HE: Constitutive phosphorylation of the FOXO1A transcription factor as a prognostic variable in gastric cancer. Mod Pathol 20: 835-842, 2007.

26. Gascoyne DM, Kypta RM and Vivanco MM: Glucocorticoids inhibit apoptosis during fibrosarcoma development by transcriptionally activating Bcl-xL. J Biol Chem 278: 18022-18029, 2003.

27. Runnebaum IB and Bruning A: Glucocorticoids inhibit cell death in ovarian cancer and up-regulate caspase inhibitor cIAP2. Clin Cancer Res 11: 6325-6332, 2005.

28. Park SY, Lee SA and Han IH: Clinical significance of metabotropic glutamate receptor 5 expression in oral squamous cell carcinoma. Oncol Rep 17: 81-87, 2007. 